\title{
Epimorphisms between Finite 4-Valued Modal Algebras
}

\author{
Aldo V. Figallo1, Elda Pick², Susana Saad ${ }^{3}$ \\ ${ }^{1}$ Instituto de Ciencias Básicas, Universidad Nacional de San Juan, San Juan, Argentina \\ ${ }^{2}$ Facultad de Ingeniería, Universidad Nacional de San Juan, San Juan, Argentina \\ ${ }^{3}$ Instituto de Ciencias Básicas, Facultad de Filosofía, Universidad Nacional de San Juan, San Juan, Argentina \\ Email: avfigallo@gmail.com, epick@unsj.edu.ar, ssaad@unsj.edu.ar
}

Received 19 December 2013; revised 19 January 2014; accepted 26 January 2014

Copyright (C) 2014 by authors and Scientific Research Publishing Inc.

This work is licensed under the Creative Commons Attribution International License (CC BY).

http://creativecommons.org/licenses/by/4.0/

c) (i) Open Access

\begin{abstract}
In this paper, we determine conditions for the existence of an epimorphism between two finite 4-valued modal algebras and state a method to obtain it. Furthermore, we obtain formulas which generalize those indicated by R. Sikorski for finite Boolean algebras [1], and by M. Abad and A. V. Figallo for finite 3-valued Lukasiewicz algebras [2].
\end{abstract}

\section{Keywords}

Four Valued Modal Algebras, Tetravalent Modal Algebras, De Morgan Algebra, Epimorphisms, Automorphisms

\section{Introduction}

Tetravalent modal algebras were first considered by A. Monteiro and studied mainly by I. Loureiro, A. V. Figallo, A. Ziliani and P. Landini (see [3]-[10]). Later, in 2000, J. M. Font and M. Rius [11] showed their interest in the logics originated by the lattice aspects of these algebras. Following A. V. Figallo's terminology, we call them four-valued modal algebras (or 4-valued modal algebras). In addition, the interesting 2013 work by M. Coniglio and M. Figallo (see [12]) has given, in our opinion, a new impulse to the profound study and development of the tetravalent modal algebras. For further information the reader interested in this subject is referred to the bibliography recommended below.

Let us now recall that

An algebra $\langle A, \wedge, \vee, \sim, 0,1\rangle$ of type $(2,2,1,0,0)$ is a De Morgan algebra [13] if $\langle A, \wedge, \vee, 0,1\rangle$ is a bounded distributive lattice with least element 0 , greatest element 1 , and $\sim$ satifies the equations $\sim x=x$ and $\sim(x \vee y)=\sim x \wedge \sim y$. 
It is known that a finite De Morgan algebra $A$ is determined by its determinant system $(\Pi(A), \Psi)$, where $\Pi(A)$ is the ordered set of all prime elements of $A$ and $\Psi: \Pi(A) \rightarrow \Pi(A)$ is an antitone involution of $\Pi(A)$. Thus, $\Psi$ has the following properties:

(i) $\Psi(\Psi(p))=p$, for each $p \in \Pi(A)$,

(ii) if $p_{1}, p_{2} \in \Pi(A)$ and $p_{1} \leq p_{2}$, then $\Psi\left(p_{2}\right) \leq \Psi\left(p_{1}\right)$.

Moreover, the operation $\sim$ is given in the following way:

$$
\sim x= \begin{cases}\vee\{p \in \Pi(A): \Psi(p) \nless x\}, & \text { if } x \neq 0, \\ 1, & \text { if } x=0 .\end{cases}
$$

In 1978 A. Monteiro defined the 4-valued modal algebras (or $M_{4}$-algebras) as algebras $\langle A, \wedge, \vee, \sim, \nabla, 0,1\rangle$ of type $(2,2,1,1,0,0)$, where $\langle A, \wedge, \vee, \sim, 0,1\rangle$ is a De Morgan algebra and the properties

$$
\sim x \vee \nabla x=1, \quad \sim x \wedge \nabla x=x \wedge \sim x
$$

are verified.

We assume the reader to be familiar with the theory of $M_{4}$-algebras as it is given in [3] [4]. In particular, in [4] is the demonstration of the following properties, which are used in this paper:

$$
x \leq \nabla x, \quad \nabla 0=0, \quad \nabla \nabla x=\nabla x, \quad \nabla(x \vee y)=\nabla x \vee \nabla y,
$$

If the operator $\Delta$ is defined by the formula $\Delta x=\sim \nabla \sim x$, then it is easy to see that $\Delta \nabla x=\nabla x$.

In what follows, we only consider finite $M_{4}$-algebras.

From [13] me have that the determinant system $(\Pi(A), \Psi)$ of an $M_{4}$-algebra $A$ has connected components of the three following types:

Type I: $\quad \because \quad$ with $\Psi(p)=p$

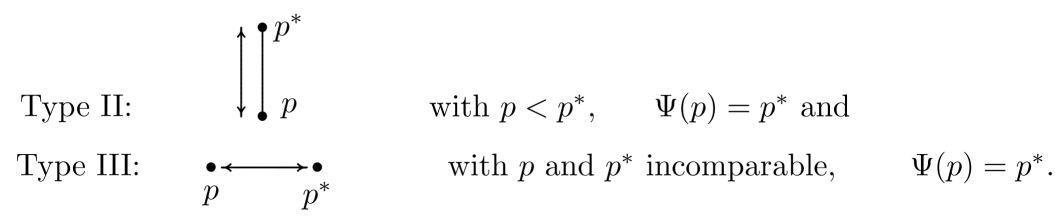

The ordered set $\Pi(A)$ has the following diagram:

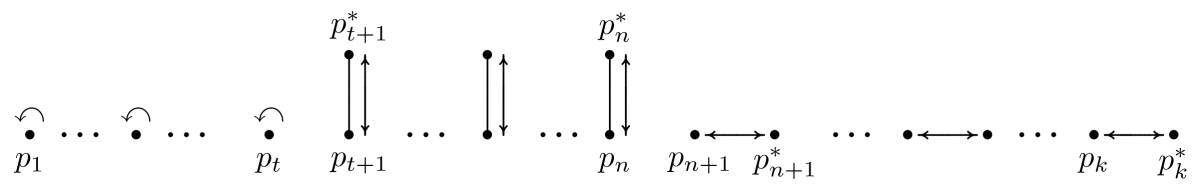

We will denote by $B(A), T(A)$, and $M(A)$ the ordered sets $\left\{p_{1}, \cdots, p_{t}\right\},\left\{p_{t+1}, p_{t+1}^{*}, \cdots, p_{n}, p_{n}^{*}\right\}$, $\left\{p_{n+1}, p_{n+1}^{*}, \cdots, p_{k}, p_{k}^{*}\right\}$, respectively.

Frequently we will write $A_{t, n-t, k-n}$ instead of $A$.

On the other hand, the operator $\nabla$ is given by the formula

$$
\nabla x= \begin{cases}\vee\{p \vee \Psi(p): p \in \Pi(A), p \leq x\}, & \text { if } x \neq 0, \\ 0, & \text { if } x=0 .\end{cases}
$$

Then,

$$
\nabla p= \begin{cases}p_{j}, & \text { if } p=p_{j} \text { and } 1 \leq j \leq t, \\ p_{j}^{*}, & \text { if } p=p_{j} \text { or } p=p_{j}^{*} \text { and } t+1 \leq j \leq n, \\ p_{j} \vee \Psi\left(p_{j}\right), & \text { if } p=p_{j} \text { or } p=p_{j}^{*} \text { and } n+1 \leq j \leq k .\end{cases}
$$

\section{2. $M_{4}$-Epimorphisms and $M_{4}$-Functions}

We will denote the sets $\Pi(A) \cup\{\nabla p: p \in \Pi(A)\}$ and $M(A) \cup\{\nabla p: p \in M(A)\}$ by $R(A)$ and $S(A)$, res- 
pectively.

The lemma given below will be used in the proof of Lemma 2.5

Lemma 2.1 If $p \in \Pi(A)$, then either $p=\nabla p$ or $\Delta p=0$

Proof. If $p \neq \nabla p$, then $p<\nabla p$, so $p=p_{j}$ with $t+1 \leq j \leq k$. Then,

$$
\begin{gathered}
\sim p_{j}=\underset{\substack{q \in \Pi(A) \\
\Psi(q) \pm p_{j}}}{\vee} q=\underset{\substack{q \in \Pi(A) \\
q \neq p_{j}^{*}}}{\bigvee} q, \\
\nabla \sim p_{j}=\nabla \underset{\substack{q \in \Pi(A) \\
q \neq p_{j}^{*}}}{\vee} q=\underset{\substack{q \in \Pi(A) \\
q \neq p_{j}^{*}}}{\bigvee} \nabla q=\underset{\substack{q \in \Pi(A) \\
q \neq p_{j}^{*}}}{\vee}(q \vee \Psi(q))=\underset{q \in \Pi(A)}{\bigvee} q=1 .
\end{gathered}
$$

Thus, $\sim \nabla \sim p_{j}=0$, that is $\Delta p_{j}=\Delta p=0$.

In what follows, we will denote with $\operatorname{Epi}\left(A, A^{\prime}\right)$ the set of all $M_{4}$-epimorphisms from the $M_{4}$-algebra $A$ into the $M_{4}$-algebra $A^{\prime}$.

Definition 2.1 A mapping $f: R\left(A^{\prime}\right) \rightarrow R(A)$ is called an $M_{4}$-function if and only if the following conditions are satisfied:

(M1) $f$ is one to one,

(M2) $f\left(\Psi\left(p^{\prime}\right)\right)=\Psi\left(f\left(p^{\prime}\right)\right)$ for all $p^{\prime} \in \Pi\left(A^{\prime}\right)$ and $f\left(p^{\prime}\right) \in \Pi\left(A^{\prime}\right)$,

(M3) $f\left(\nabla q^{\prime}\right)=\nabla\left(f\left(q^{\prime}\right)\right)$ for all $q^{\prime} \in R\left(A^{\prime}\right)$.

We will denote by $\mathcal{F}\left(A^{\prime}, A\right)$ the set of all $M_{4}$-functions from $R\left(A^{\prime}\right)$ into $R(A)$.

Lemma 2.2 If $f \in \mathcal{F}\left(A^{\prime}, A\right), A_{x}^{*}=\left\{q^{\prime} \in R\left(A^{\prime}\right): f\left(q^{\prime}\right) \leq x\right\}$ and $A_{x}^{\prime}=\left\{p^{\prime} \in \Pi\left(A^{\prime}\right): f\left(p^{\prime}\right) \leq x\right\}$, then:

(i) $f$ is an isotone map,

(ii) $\vee\left\{q^{\prime}: q^{\prime} \in A_{x}^{*}\right\}=\bigvee\left\{p^{\prime}: p^{\prime} \in A_{x}^{\prime}\right\}$.

Proof.

(i) If $q_{1}^{\prime}, q_{2}^{\prime} \in R\left(A^{\prime}\right)$ and $q_{1}^{\prime}<q_{2}^{\prime}$, then $\nabla q_{1}^{\prime}=q_{2}^{\prime}$. Thus, $f\left(q_{1}^{\prime}\right) \leq \nabla f\left(q_{1}^{\prime}\right)=f\left(\nabla q_{1}^{\prime}\right)=f\left(q_{2}^{\prime}\right)$, and by (M1) we obtain $f\left(q_{1}^{\prime}\right)<f\left(q_{2}^{\prime}\right)$.

(ii) Let $N_{x}=A_{x}^{*} \backslash A_{x}^{\prime}, \quad a=\bigvee\left\{p^{\prime}: p^{\prime} \in A_{x}^{\prime}\right\}, \quad b=\bigvee\left\{q^{\prime}: q^{\prime} \in A_{x}^{*}\right\}, \quad c=\bigvee\left\{r^{\prime}: r^{\prime} \in N_{x}\right\}$. It is easy to see that $b=a \vee c$. We shall show that $c \leq a$. Indeed, if $r^{\prime} \in N_{x}$, then there exists $p_{o}^{\prime} \in \Pi\left(A^{\prime}\right)$ such that $r^{\prime}=p_{o}^{\prime} \vee \Psi\left(p_{o}^{\prime}\right)$. Hence, $p_{o}^{\prime} \leq r^{\prime}$ and $\psi\left(p_{o}^{\prime}\right) \leq r^{\prime}$. Since $f\left(r^{\prime}\right) \leq x$, we obtain $f\left(p_{o}^{\prime}\right) \leq x$ and $f\left(\Psi\left(p_{o}^{\prime}\right)\right) \leq x$, with $p_{o}^{\prime}, \Psi\left(p_{o}^{\prime}\right) \in \Pi(A)$, that is $p_{o}^{\prime}, \Psi\left(p_{o}^{\prime}\right) \in A_{x}^{\prime}$. Hence, $r^{\prime} \leq a$; thus, $c \leq a$. Finally, $b=a$.

Definition 2.2 Let $f \in\left(A^{\prime}, A\right) . F: A \rightarrow A^{\prime}$ is an $M$-function associated with $f$ if and only if $F$ is defined by:

$$
F(x)= \begin{cases}\vee\left\{q^{\prime}: q^{\prime} \in A_{x}^{*}\right\}, & \text { if } A_{x}^{*} \neq \varnothing, \\ 0, & \text { if } A_{x}^{*}=\varnothing .\end{cases}
$$

We denote by $\mathcal{M}\left(A, A^{\prime}\right)$ the set of all $M$-functions.

The following lemma is used to prove that every $M$-function is an epimorphism.

Lemma 2.3

(I) If $p^{\prime} \in \Pi\left(A^{\prime}\right)$ and $f\left(\Psi\left(p^{\prime}\right)\right) \nless x$, then $\Psi\left(p^{\prime}\right) \nless F(x)$.

(II) If $p \in \Pi(A)$, then $F(p)=0$ or $F(p) \in \Pi\left(A^{\prime}\right)$.

(III)If $p \in \Pi(A)$, then $F(\nabla p)=\nabla F(p)$.

Proof.

(I) Assume that $\Psi\left(p^{\prime}\right) \leq F(x)$. Since $F(x)=\bigvee\left\{s^{\prime}: s^{\prime} \in A_{x}^{\prime}\right\}$ and $\Psi\left(p^{\prime}\right) \in \Pi\left(A^{\prime}\right)$, it follows that there exists $s_{o}^{\prime} \in A_{x}^{\prime}$, such that $\Psi\left(p^{\prime}\right) \leq s_{o}^{\prime}$. From this we obtain $f\left(\Psi\left(p^{\prime}\right)\right) \leq f\left(s_{o}^{\prime}\right)$; as a consequence, $f\left(\Psi\left(p^{\prime}\right)\right) \leq x$, which contradicts the hypothesis.

(II) Suppose $F(p) \neq 0$. There are two possibilities for $p$ :

(a) $p$ is minimal in $\Pi(A)$. As $F(p) \neq 0, A_{p}^{\prime} \neq \varnothing$, then there exists $p^{\prime} \in \Pi\left(A^{\prime}\right)$ such that $f\left(p^{\prime}\right) \leq p$.

Since $f\left(p^{\prime}\right) \in \Pi(A)$ and $p$ is minimal, then $f\left(p^{\prime}\right)=p$ must hold, and from (M1), $A_{p}^{\prime}=\left\{p^{\prime}\right\}$ results. Thus, $F(p)=p^{\prime}$ with $f\left(p^{\prime}\right)=p$.

(b) $p$ is maximal and not minimal in $\Pi(A)$. Then, $p=\nabla q$ with $q \in \Pi(A)$ and $q<p$. From $F(p) \neq 0$ 
there exists $p^{\prime} \in \Pi\left(A^{\prime}\right)$ such that $f\left(p^{\prime}\right) \leq p$. If $F(q)=0$ we get $f\left(p^{\prime}\right)=p$, and then $F(p)=p^{\prime}$. If $F(q) \neq 0$, as in (a), there exists a unique $q^{\prime} \in \Pi\left(A^{\prime}\right)$ such that $f\left(q^{\prime}\right)=q$. Moreover, (i) $q^{\prime}<\nabla q^{\prime}$ because if $q^{\prime}=\nabla q^{\prime}$, then $f\left(q^{\prime}\right)=f\left(\nabla q^{\prime}\right)=\nabla f\left(q^{\prime}\right)=\nabla q=p$, which is not possible. Consequently, (ii) $f\left(q^{\prime}\right)<f\left(\nabla q^{\prime}\right)$.

From (i) it follows that $q^{\prime}$ is Type II or Type III; in the latter case $\Psi\left(q^{\prime}\right)<\nabla q^{\prime}$ and therefore $p=\Psi(q)=\Psi\left(f\left(q^{\prime}\right)\right)=f\left(\Psi\left(q^{\prime}\right)\right)<f\left(\nabla q^{\prime}\right)=\nabla f\left(q^{\prime}\right)=\nabla q=p$, which is not possible. Then, $q^{\prime}$ is Type II, so that $\Psi\left(q^{\prime}\right)=\nabla q^{\prime}$, then $\nabla q^{\prime} \in \Pi\left(A^{\prime}\right)$, and thus (iii) $f\left(\nabla q^{\prime}\right) \in \Pi\left(A^{\prime}\right)$. From (ii) and (iii) it results $f\left(\nabla q^{\prime}\right)=p$. Therefore, $A_{p}^{\prime}=\left\{\nabla q^{\prime}\right\}$, so $F(p)=\nabla q^{\prime}$.

(III) Let $p \in \Pi(A)$.

(a) If $F(p) \neq 0$, then from (II) $F(p)=p^{\prime}$, with $f\left(p^{\prime}\right)=p$. Thus, $f\left(\nabla p^{\prime}\right)=\nabla f\left(p^{\prime}\right)=\nabla p$; then, $F(\nabla p)=\nabla p^{\prime}=\nabla F(p)$.

(b) If $F(p)=0$, then $\nabla F(p)=\nabla 0=0$. There are two cases:

(b) $p=\nabla p$. Then, $F(\nabla p)=F(p)=0=\nabla F(p)$.

$\left(\mathrm{b}_{2}\right) \quad p<\nabla p$ and $F(\nabla p) \neq 0$, then:

$\left(\mathrm{b}_{21}\right)$ if $\nabla p \in \Pi(A)$, then $F(\nabla p)=p^{\prime}$ with $f\left(p^{\prime}\right)=\nabla p$, and $f\left(\nabla p^{\prime}\right)=\nabla f\left(p^{\prime}\right)=\nabla \nabla p=\nabla p=f\left(p^{\prime}\right)$; therefore, by (M1) $\nabla p^{\prime}=p^{\prime}$. Moreover, it is clear that $p^{\prime}$ is a minimal prime, because if $q^{\prime}<p^{\prime}$, then $f\left(q^{\prime}\right)<f\left(p^{\prime}\right)=\nabla p$, which is not possible. Then, $\nabla p^{\prime}=p^{\prime}$ is minimal, and as a consequence, $p^{\prime}=\Psi\left(p^{\prime}\right)$. Thus, $\nabla p=f\left(p^{\prime}\right)=f\left(\Psi\left(p^{\prime}\right)\right)=\Psi\left(f\left(p^{\prime}\right)\right)=\Psi(\nabla p)$, which is a contradiction. Then, $F(\nabla p)=0$ holds; therefore, $F(\nabla p)=\nabla F(p)$

$\left(\mathrm{b}_{22}\right)$ if $\nabla p \notin \Pi(A)$, then $p=f\left(p^{\prime}\right)$ is Type III; therefore, $F(p)=p^{\prime}$ and $F(\nabla p)=p^{\prime} \vee \Psi\left(p^{\prime}\right)=\nabla p^{\prime}=\nabla F(p)$.

Lemma 2.4 If $\mathcal{F}\left(A^{\prime}, A\right) \neq \varnothing$, then $\mathcal{M}\left(A, A^{\prime}\right) \subseteq \operatorname{Epi}\left(A, A^{\prime}\right)$.

Proof. Let $f \in \mathcal{F}\left(A^{\prime}, A\right)$ and $F \in \mathcal{M}\left(A, A^{\prime}\right)$ be the $M$-function associated by $f$.

(a) It is obvious that $F(1)=1$ and $F(0)=0$.

(b) $F(x \vee y)=F(x) \vee F(y)$. Actually, it is easy to see that $A_{x}^{\prime} \cup A_{y}^{\prime} \subseteq A_{x \vee y}^{\prime}$; moreover, $p^{\prime} \in A_{x \vee y}^{\prime}$ if and only if $f\left(p^{\prime}\right) \leq x \vee y$. Since $f\left(p^{\prime}\right) \in \Pi\left(A^{\prime}\right), f\left(p^{\prime}\right) \leq x$ or $f\left(p^{\prime}\right) \leq y$ must hold; hence, $f\left(p^{\prime}\right) \in A_{x}^{\prime}$ or $f\left(p^{\prime}\right) \in A_{y}^{\prime}$, that is $p^{\prime} \in A_{x}^{\prime} \cup A_{y}^{\prime}$; then $A_{x \vee y}^{\prime}=A_{x}^{\prime} \cup A_{y}^{\prime}$. Finally,

$$
F(x \vee y)=\vee A_{x \vee y}^{\prime}=\vee\left(A_{x}^{\prime} \cup A_{y}^{\prime}\right)=\vee\left\{r^{\prime}: f\left(r^{\prime}\right) \leq x \text { or } f\left(r^{\prime}\right) \leq y\right\}=F(x) \vee F(y) .
$$

(c) $F(\sim x)=\sim F(x)$ for all $x \in A$. Indeed,

(c) $F(\sim x) \leq \sim F(x)$.

It is sufficient to show that, if $q^{\prime} \in A_{\sim x}^{\prime}$, then $q^{\prime} \leq \sim F(x)$.

If $q_{o}^{\prime} \in A_{\sim x}^{\prime}$, then $q_{o}^{\prime} \in \Pi\left(A^{\prime}\right)$ and $f\left(q_{o}^{\prime}\right) \leq \sim x=\vee\{p \in \pi(A): \Psi(p) \nless x\}$. Hence, there exists $p_{o} \in \Pi(A)$ such that $f\left(q_{o}^{\prime}\right) \leq p_{o}$, and $\Psi\left(p_{o}\right) \leq x_{o}$. Then, $\Psi\left(p_{o}\right) \leq \Psi\left(f\left(q_{o}^{\prime}\right)\right)$ holds. Taking into account that $f\left(\Psi\left(q_{o}^{\prime}\right)\right)=\Psi\left(f\left(q_{o}^{\prime}\right)\right)$, by Lemma 2.3(I), we have that $\Psi\left(q_{o}^{\prime}\right) \leq F(x)$; then $q_{o}^{\prime} \leq \sim F(x)$.

$\left(\mathrm{c}_{2}\right) \sim F(x) \leq F(\sim x)$.

Let $p^{\prime} \in \Pi(A)$. The proof is a consequence of the fact that the following conditions are pairwise equivalent:
(1) $p^{\prime} \leq \sim F(x)$
(2) $\Psi\left(p^{\prime}\right) \not F(x)$
(3) $f\left(\Psi\left(p^{\prime}\right)\right) \not x$
(4) $\Psi\left(f\left(p^{\prime}\right)\right) \not x$
(5) $f\left(p^{\prime}\right) \leq \sim x$
(6) $p^{\prime} \leq F(\sim x)$

(d) $F(\nabla x)=\nabla F(x)$, for all $x \in A$, which is inmediate consequence of (III), $\nabla(x \vee y)=\nabla x \vee \nabla y$ and (b).

(e) $F$ is clearly onto.

Lemmas 2.5 and 2.6 are necessary to show that all $M_{4}$-epimorphisms are an $M_{4}$-function $\mathrm{M}$.

Lemma 2.5 If $h \in \operatorname{Epi}\left(A, A^{\prime}\right)$ and $q^{\prime} \in R\left(A^{\prime}\right)$, then there exists a unique $q \in R(A)$ such that $h(q)=q^{\prime}$.

Proof.

(1) (a) If $q^{\prime} \in \Pi\left(A^{\prime}\right)$, then there is $q \in \Pi(A)$ such that $h(q)=q^{\prime}$. Indeed, let $q^{\prime} \in \Pi\left(A^{\prime}\right)$; since $h$ is onto, there exists $x \in A$ such that $h(x)=q^{\prime}$; therefore $h^{-1}\left(\left\{q^{\prime}\right\}\right) \neq \varnothing$, and then, $h^{-1}\left(\left\{q^{\prime}\right\}\right)=\left\{x_{1}, \cdots, x_{l}\right\}$. If $q=\bigwedge_{i=1}^{l} x_{i}$, then, $h(q)=q^{\prime}$ and $q \neq 0$. If $q=y \vee z$, then $y \leq q, \quad z \leq q$ and $q^{\prime}=h(q)=h(y) \vee h(z)$; since 
$q^{\prime} \in \Pi\left(A^{\prime}\right), \quad q^{\prime}=h(y)$ or $q^{\prime}=h(z)$ must hold. If $q^{\prime}=h(y)$, then $y \in h^{-1}\left(\left\{q^{\prime}\right\}\right)$; therefore $y=x_{j}$ for some $j \in\{1, \cdots, l\}$; hence, $q \leq y$ and so $q=y$. Then $q=y$ or $q=z$. Thus, $q \in \Pi(A)$.

(b) If $q \in \Pi(A)$ and $h(q)=q^{\prime}$, then $q$ is unique. Indeed, if $p \in h^{-1}\left(\left\{q^{\prime}\right\}\right) \cap \Pi(A)$, then $p \in \Pi(A)$ and $h(p)=q^{\prime}$. Accordlingly, $q$ is the smallest $r \in \Pi(A)$ such that $h(r)=q^{\prime}, q \leq p$. If $q<p$, then $p=\nabla q$; by Lemma 2.1, $\Delta q=0$ must hold. Furthermore, $q^{\prime}=h(p)=h(\nabla p)=\nabla h(p)=\nabla q^{\prime}$, and then $\Delta q^{\prime}=\Delta h(p)=\Delta \nabla q^{\prime}=\nabla q^{\prime}$. On the other hand, $\Delta q^{\prime}=\Delta h(q)=h(\Delta q)=0$, which is a contradiction.

(2) If $q^{\prime} \in R\left(A^{\prime}\right) \backslash \Pi\left(A^{\prime}\right)$, then there is $p^{\prime} \in \Pi\left(A^{\prime}\right)$ such that $q^{\prime}=p^{\prime} \vee \Psi\left(p^{\prime}\right)$; by 1 . there is a unique $p \in \Pi(A)$ such that $h(p)=p^{\prime}$. Let $q=p \vee \Psi(p)$; it is easy to prove that $p$ and $p^{\prime}$ are of the same type. So $q^{\prime}=\nabla p^{\prime}=\nabla h(p)=h(\nabla p)=h(p \vee \Psi(p))=h(p) \vee h(\Psi(p))$.

In addition, the following holds: $q^{\prime}=\nabla p^{\prime}=p^{\prime} \vee \Psi\left(p^{\prime}\right)=h(p) \vee \Psi(h(p))$, giving the result that follows:

$$
h(p) \vee h(\Psi(p))=h(p) \vee \Psi(h(p))
$$

Moreover, $h(p) \wedge \Psi(h(p))=p^{\prime} \wedge \Psi\left(p^{\prime}\right)=0 ; h(p) \wedge h(\Psi(p))=h(p \wedge \Psi(p))=0$; then

$$
h(p) \wedge h(\Psi(p))=h(p) \wedge \Psi(h(p))
$$

From (i) and (ii), it follows that $h(\Psi(p))=\Psi(h(p))$. It is evident that $q \in R(A)$ is unique and $h(q)=q^{\prime}$.

Definition 2.3 Let $h \in \operatorname{Epi}\left(A, A^{\prime}\right)$. The function $f: R\left(A^{\prime}\right) \rightarrow R(A)$ is induced by $h$ if $f$ is defined by $f\left(q^{\prime}\right)=q$, if and only if $h(q)=q^{\prime}$.

Lemma 2.6 Every function induced by an $M_{4}$-epimorphism is an $M_{4}$-function.

Proof. $f$ be the function induced by the $M_{4}$-epimorphism $h$.

(M1) It is easy to prove that $f$ is one to one.

(M3) Let $f\left(p^{\prime}\right)=p$, with $p^{\prime}=h(p)$. Therefore, $h(\nabla p)=\nabla h(p)=\nabla p^{\prime}$; consequently, $f\left(\nabla p^{\prime}\right)=\nabla p$, i.e. $f\left(\nabla p^{\prime}\right)=\nabla f\left(p^{\prime}\right)$.

As a consequence of (M1) and (M3), $f$ is an order-preserving function.

(M2) Let $p^{\prime} \in \Pi\left(A^{\prime}\right), \quad f\left(p^{\prime}\right)=q$ with $h(q)=p^{\prime}$.

(1) If $p^{\prime}$ is Type I, then $p^{\prime}=\Psi\left(p^{\prime}\right)=\nabla p^{\prime}$, and

(a) if $q<\Psi(q)$, since $h(q) \in \Pi(A)$ and $h$ is one to one on the set of all prime $p$ that $h(p) \neq 0$, $h(q)<h(\Psi(q))$ must hold; then $p^{\prime}=h(q)<h(\Psi(q))=h(\nabla q)=\nabla h(q)=\nabla p^{\prime}$, which is a contradiction.

(b) if $\Psi(q)<q$, then $h(\Psi(q)) \leq h(q)=p^{\prime}$. If $h(\Psi(q))=p^{\prime}$, then $f\left(p^{\prime}\right)=\Psi(q)$; since $\mathrm{f}$ es one to one must be $q=\Psi(q)$, which is a contradiction. So $h(\Psi(q))<p^{\prime}$ and consequently $h(\Psi(q))=0$ and then $p^{\prime}=\nabla p^{\prime}=\nabla h(q)=h(\nabla q)=h(\nabla \Psi(q))=\nabla h(\Psi(q))=\nabla 0=0$, which is not possible.

This shows that in this case $\Psi(q) \nless q$ and $q \nless \Psi(q)$; as a result $\Psi\left(f\left(p^{\prime}\right)\right)=f\left(p^{\prime}\right)$, so that either $\Psi\left(f\left(p^{\prime}\right)\right)=f\left(\Psi\left(p^{\prime}\right)\right)$, or $\Psi(q)$ and $q$ are not comparable, which is not possible since $q=f\left(p^{\prime}\right)=f\left(\nabla p^{\prime}\right)=\nabla f\left(p^{\prime}\right)=\nabla q$.

(2) If $p^{\prime}$ is Type II, then either $p^{\prime}<\Psi\left(p^{\prime}\right)$ or $\Psi\left(p^{\prime}\right)<p^{\prime}$. In the first case $f\left(p^{\prime}\right)<f\left(\Psi\left(p^{\prime}\right)\right)$ and therefore $\Psi\left(f\left(p^{\prime}\right)\right)=f\left(\Psi\left(p^{\prime}\right)\right)$. A similar argument is valid if $\Psi\left(p^{\prime}\right)<p^{\prime}$.

(3) If $p^{\prime}$ is Type III, then $f\left(p^{\prime}\right)$ is Type III, and it is not comparable with $f\left(\Psi\left(p^{\prime}\right)\right)$; consequently, $f\left(\Psi\left(p^{\prime}\right)\right)=\Psi\left(f\left(p^{\prime}\right)\right)$.

Lemma 2.7 $\operatorname{Epi}\left(A, A^{\prime}\right) \subseteq \mathcal{M}\left(A, A^{\prime}\right)$.

Proof. If $\operatorname{Epi}\left(A, A^{\prime}\right)=\varnothing$, the proof is obvious. Let $h \in \operatorname{Epi}\left(A, A^{\prime}\right), f$ the function induced by $h$, and $F$ the $M$-function associated to $f$. It is clear that $h(0)=0=F(0)$. Let $x \in A, x \neq 0$. Then, $x=\bigvee\{p: p \in \Pi(A), p \leq x\}$ and consequently, $h(x)=\bigvee\{h(p): p \in \Pi(A), p \leq x\}$. For each $p \in \Pi(A)$, $p \leq x$, let $p^{\prime}=h(p) \in \Pi\left(A^{\prime}\right)$. Then $h(x)=\bigvee\left\{p^{\prime}: p^{\prime} \in \Pi\left(A^{\prime}\right), f\left(p^{\prime}\right) \leq x\right\}=\bigvee\left\{p^{\prime}: p^{\prime} \in A_{x}^{\prime}\right\}=F(x)$. Thus, $h=F$; as a consequence, $h \in \mathcal{M}\left(A, A^{\prime}\right)$.

Teorema 2.1 $\operatorname{Epi}\left(A, A^{\prime}\right)=\mathcal{M}\left(A, A^{\prime}\right)$.

Proof. It follows from Lemmas 2.4 and 2.7.

Teorema 2.2 $\operatorname{Epi}\left(A, A^{\prime}\right)|=| \mathcal{F}\left(A^{\prime}, A\right) \mid$.

Remarks 2.1 Let $A=A_{t, m-t, k-m}, \quad A^{\prime}=A_{s, n-s, r-n}$. Then,

(i) $F\left(A^{\prime}, A\right) \neq \varnothing$ if only if $s \leq t, n-s \leq m-t$, and $r-n \leq k-m$. 
(ii) The following holds

$\left|B_{1}\left(A^{\prime}, A\right)\right|=V_{t}^{s}, \quad\left|T_{1}\left(A^{\prime}, A\right)\right|=V_{m-t}^{n-s}, \quad\left|S_{1}\left(A^{\prime}, A\right)\right|=\prod_{i=0}^{r-n-1}(2 \cdot(k-m)-2 \cdot i)$,

where $V_{p}^{q}= \begin{cases}\frac{p !}{(p-q) !} & \text { if } p>q \text {, } \\ 0, & \text { otherwise, }\end{cases}$

$B_{1}\left(A^{\prime}, A\right)$ is the set of all injective functions $f \in B(A)^{B\left(A^{\prime}\right)}, T_{1}\left(A^{\prime}, A\right)$ is the set of all injective functions $f \in T(A)^{T\left(A^{\prime}\right)}$ such that $f\left(\Psi\left(p^{\prime}\right)\right)=\Psi\left(f\left(p^{\prime}\right)\right)$ for each $p \in T(A), S_{1}\left(A^{\prime}, A\right)$ is the set of all injective functions $f \in S(A)^{S\left(A^{\prime}\right)}$ such that $f\left(\Psi\left(p^{\prime}\right)\right)=\Psi\left(f\left(p^{\prime}\right)\right)$ for all $p^{\prime} \in M\left(A^{\prime}\right)$, and $f\left(\nabla\left(q^{\prime}\right)\right)=\nabla f\left(q^{\prime}\right)$ for all $q^{\prime} \in S\left(A^{\prime}\right)$.

Teorema 2.3 If $1 \leq s \leq t, 1 \leq n-s \leq m-t$, and $1 \leq r-n \leq k-m$, then

$$
\left|\operatorname{Epi}\left(A_{t, m-t, k-m}, A_{s, n-s, r-n}^{\prime}\right)\right|=2^{r-n} \cdot V_{t}^{s} \cdot V_{m-t}^{n-s} \cdot \prod_{i=0}^{r-n-1}(k-m-i)
$$

Proof. If $f \in \mathcal{F}\left(A^{\prime}, A\right), f_{1}=f_{\mid B\left(A^{\prime}\right)}, \quad f_{2}=f_{\mid T\left(A^{\prime}\right)}$ and $f_{3}=f_{\mid S\left(A^{\prime}\right)}$, then the map $f \rightarrow\left(f_{1}, f_{2}, f_{3}\right)$ is a bijective correspondence between $F\left(A^{\prime}, A\right)$ and $B_{1}\left(A^{\prime}, A\right) \times T_{1}\left(A^{\prime}, A\right) \times S_{1}\left(A^{\prime}, A\right)$. From this, Theorem 2.1 and Remarks 2.1 this theorem follows.

An immediate consequence of theorem 2.3 is

Teorema $2.4\left|\operatorname{Aut}\left(A_{t, m-t, k-m}\right)\right|=2^{k-m} \cdot t ! \cdot(m-t) ! \cdot(k-m) !$

\section{References}

[1] Sikorski, R. (1949) On the Inducing of Homomorphisms by Mappings. Fundamenta Mathematicae, 36, 7-22.

[2] Abad, M. and Figallo, A.V. (1992) On the Lukasiewicz homomorphisms. Instituto de Ciencias Básicas, UNSJ, 1-12.

[3] Loureiro, I. (1982) Axiomatisation et proprietes des algèbres modales tetravalentes. C.R. Acad. Sc. Paris, t. 295, Serie I, 555-557.

[4] Loureiro, I. (1984) Finite Tetravalent Modal Algebras. Revista de la Unión Matemática Argentina, 31, 187-191.

[5] Loureiro, I. (1983) Prime Spectrum of a Tetravalent Modal Algebras. Notre Dame Journal of Formal Logic, 24, 389394. http://dx.doi.org/10.1305/ndjfl/1093870382

[6] Figallo, A.V. (1990) Notes on Generalized N-Lattices. Revista de la Unión Matemática Argentina, 35, 61-65.

[7] Figallo, A.V. (1992) On the Congruence in Four-Valued Modal Algebras. Portugaliae Mathematica, 49, $249-261$.

[8] Figallo, A.V. and Ziliani, A. (1991) Symmetric Tetra-Valued Modal Algebras. Notas de la Sociedad Matemática de Chile, 10, 133-141.

[9] Ziliani, A. (1995) On Axioms and Properties of Monadic Four-Valued Algebras. Actas del Tercer Congreso Dr. A.R.R. Monteiro, Instituto de Matemática, U. N. del Sur, Argentina, 69-78.

[10] Figallo, A.V. and Landini, P. (1995) On Generalized I-Algebras and Modal 4-Valued Algebras. Reports on Mathematical Logic, 29, 3-18.

[11] Font, J.M. and Rius, M. (2000) An Abstract Algebraic Logic Approach to Tetravalent Modal Logics. Journal of Symbolic Logic, 65, 481-518. http://dx.doi.org/10.2307/2586552

[12] Coniglio, M.E. and Figallo, M. (2013) Hilbert-Style Presentations of Two Logics Associated to Tetravalent Modal Algebras. Studia Logica, 102, 525-539. http://dx.doi.org/10.1007/s11225-013-9489-0

[13] Monteiro, A. (1960) Matrices de Morgan Caracteristiques Pour le Calcul Propositionnel Classique. Anais da Academia Brasileira de Ciências, 52, 1-7.

\section{AMS 2000 Subject Classification}

Primary 08A35, 06D30. Secondary 03G25. 